\title{
Energy Harvesting Communications with Hybrid Energy Storage and Processing Cost
}

\author{
Omur Ozel Khurram Shahzad Sennur Ulukus \\ Department of Electrical and Computer Engineering \\ University of Maryland College Park, MD 20742 \\ omur@umd.edu_kshahzad@umd.edu_ulukus@umd.edu
}

\begin{abstract}
We consider data transmission with an energy harvesting transmitter with non-negligible processing circuitry power and a hybrid energy storage unit composed of an ideal super-capacitor (SC) and an inefficient battery. The SC has finite space for energy storage while the battery has unlimited space. The transmitter stores the harvested energy either in the SC or in the battery and the energy is drained from the SC and the battery simultaneously. In this setting, we address the offline throughput maximization problem over a point-to-point channel. We show that the solution is obtained by a sequential application of the directional glue-pouring algorithm.
\end{abstract}

\section{INTRODUCTION}

Hybrid energy storage units have been extensively used in energy harvesting communication systems, see e.g., [1] and references therein. Such storage units are obtained by augmenting a super-capacitor (SC) to the battery. In general, SCs ideally store energy; however, they suffer from low energy storage capacities. On the other hand, batteries have large storage capacities while they suffer from inefficient energy storage. In data transmission with such an energy storage unit, the transmitter has to decide the portions of the incoming energy to be saved in the SC and the battery. While it is desirable to save energy in the SC due to its perfect storage efficiency, the storage capacity limitation necessitates careful energy management. In this paper, we address this problem in an offline setting.

Offline throughput maximization for energy harvesting systems has recently been studied in various models [2]-[16]. In [2], the transmission completion time minimization problem is solved in energy harvesting systems with an unlimited capacity battery over a static channel. This line has later been extended for a finite capacity battery [3], fading channel [4], broadcast channel [5], [6], multiple access channel [7], interference channel [8] and relay channel [9], [10]. Offline throughput maximization for energy harvesting systems with leakage in energy storage was studied in [11]. In [12], offline optimal performance limits of multi-user wireless systems with energy transfer are studied. This line of research has also been extended in [13], [14] for systems with processing costs. [15] considers offline throughput maximization for energy harvesting devices in the presence of energy storage losses. In

This work was supported by NSF Grants CNS 09-64632, CCF 09-64645, CCF 10-18185 and CNS 11-47811. our recent work [16], we considered throughput maximization for energy harvesting transmitters with hybrid energy storage.

In this paper, we extend our work in [16] to address the offline throughput maximization problem for the specified hybrid energy storage model when there is additional processing cost. As emphasized in [2]-[16], energy arrivals impose causality constraints on the energy management policy. In addition, battery limitation imposes no-energy-overflow constraints [3], [4], [6]. In the presence of hybrid energy storage, the energy causality and no-energy-overflow constraints take a new form. Moreover, a time-linear additive processing cost is present in the data transmission circuitry. We model the processing cost as an additional power expenditure and the storage efficiency as a constant $\eta$, and solve for the optimal throughput.

It is well-known that circuit power consumption is nonnegligible compared to the power spent for data transmission in small scale and short range applications [17]. We note that a considerable portion of energy harvesting communication applications falls into this category, and the effects of circuit power have been investigated in previous works on energy harvesting communications [13], [14], [18], [19]. Among these works, the framework that is most pertinent to ours has been proposed in [14]. In contrast to [14], in our case, the transmitter has to additionally decide the portions of the energy cost drained from the SC and the battery in the presence of hybrid energy storage. Despite this additional complexity, we show that the solution of the throughput maximization problem with hybrid energy storage is obtained by a sequential application of an extended version of the directional glue pouring algorithm in [14]. To this end, we first construct an equivalent single epoch problem by introducing new time and power variables. In particular, we divide the available time for the SC and the battery and enforce SC and the battery to pay the energy costs in the corresponding time intervals. We show that this specific scheme yields a jointly optimal transmission and energy cost drainage scheme. We, then, generalize the single epoch analysis to multiple epochs and obtain an extension of the framework in [14] to the case of hybrid energy storage.

\section{SySTEM MODEL}

We consider a single-user additive Gaussian noise channel with an energy harvesting transmitter. The transmitter has three queues: a data queue and two energy queues. Two energy 


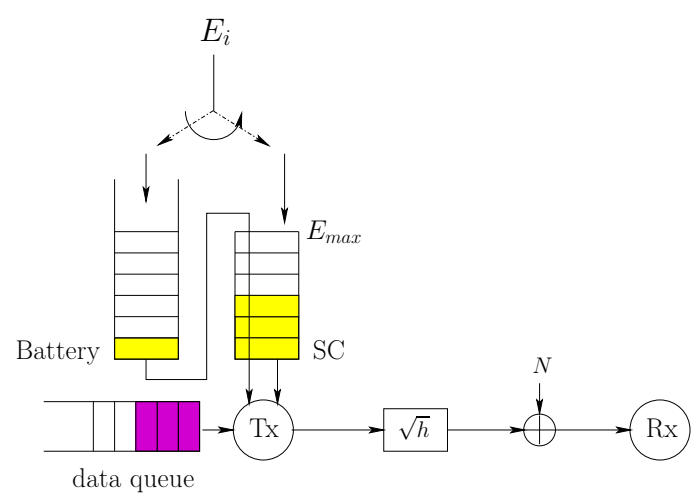

Fig. 1. System model with hybrid energy storage.

queues correspond to a hybrid energy storage unit composed of a battery and a SC as shown in Fig. 1. The battery has unlimited storage capacity whereas $\mathrm{SC}$ can store at most $E_{\max }$ units of energy. The battery is inefficient in the sense that the energy that can be drained from it is less than the amount that is stored; the SC is perfectly efficient. We assume an infinite backlog in the data queue.

The physical layer is an AWGN channel with the inputoutput relation $Y=\sqrt{h} X+N$ where $h$ is the squared channel gain and $N$ is Gaussian noise with zero-mean and unit-variance. Without loss of generality, we set $h=1$. We follow a continuous time model and instantaneous rate is

$$
r(t)=\frac{1}{2} \log (1+p(t))
$$

At time $t_{i}^{e}, E_{i}$ amount of energy arrives. $E_{0}^{b}$ and $E_{0}^{s c}$ amounts of energies are available at the beginning in the battery and in the SC, respectively. In the following, we refer to the time interval between two energy arrivals as an epoch. More specifically, epoch $i$ is the time interval $\left[t_{i}^{e}, t_{i+1}^{e}\right)$ and the length of the epoch $i$ is $\ell_{i}=t_{i+1}^{e}-t_{i}^{e}$.

Whenever energy $E_{i}$ arrives at time $t_{i}^{e}$, the transmitter stores $E_{i}^{b}$ amount in the battery and $E_{i}^{s c}=E_{i}-E_{i}^{b}$ amount in the SC. Since SC can store at most $E_{\max }$ units of energy, $E_{i}^{s c}$ must be chosen such that no energy unnecessarily overflows. For this reason, $E_{i}^{s c} \leq E_{\max }$ must necessarily be satisfied. The efficiency of the battery is given by the parameter $\eta$ where $0 \leq$ $\eta<1$ : If $E_{i}^{b}$ units of energy is stored in the battery, then $\eta E_{i}^{b}$ units can be drained and $(1-\eta) E_{i}^{b}$ units are lost. Moreover, we assume that the available energy in the battery can be transferred to the SC instantaneously ${ }^{1}$. As a consequence, none of the arrived energy overflows; however, there is an energy loss due to the inefficiency of the battery.

Moreover, we note that the power policy should cause no energy overflow in the SC. In order to express this constraint, we divide each incremental drained energy $p(u) d u$ as a linear combination of the energy drained from the $\mathrm{SC}, p^{s c}(u) d u$, and the energy drained from the battery, $p^{b}(u) d u$. That is, $p(u) d u=p^{s c}(u) d u+p^{b}(u) d u$. We are allowed to divide

\footnotetext{
${ }^{1}$ In real systems, switching time between the battery and the SC is very small compared to epoch lengths of interest [1].
}

$p(u) d u$ into such components since the energy in the battery can be instantaneously transferred to the SC. The transmitter's circuitry causes an additive time-linear processing cost in data transmission. In particular, the processing cost could be viewed as a constant circuit power $\epsilon$ whenever it is active. Hence, for a transmit power policy $p(t)$, the total power consumption is $p(t)+\epsilon \mathbf{1}_{p(t)>0}$ where $\epsilon$ is in energy units per time units. In this case, the energy causality and no-energy-overflow constraints can be expressed as:

$$
\begin{array}{r}
\int_{0}^{t_{i}^{e}}\left(p(u)+\epsilon \mathbf{1}_{p(u)>0}\right) d u \leq \sum_{j=0}^{i-1} E_{j}^{s c}+\eta E_{j}^{b}, \quad \forall i \\
\sum_{j=0}^{i} E_{j}^{s c}-\int_{0}^{t_{i}^{e}}\left(p^{s c}(u)+\epsilon^{s c} \mathbf{1}_{p(u)>0}\right) d u \leq E_{\max }, \quad \forall i
\end{array}
$$

where $\epsilon_{i}^{s c}$ and $\epsilon_{i}^{b}$ are the portions of the processing power drained from the SC and the battery, respectively, in epoch $i$ : $\epsilon=\epsilon_{i}^{s c}+\epsilon_{i}^{b}$.

\section{OfFline ThroughPUT MAXIMIZATION}

\section{A. The Case of a Single Epoch}

We start by considering the single epoch case. Assume that $E^{s c}$ and $E^{b}$ units of energy are available at $t=0$, respectively, and let the deadline be set to infinity. We have the following optimization problem:

$$
\max _{t, p^{s c}(.), p^{b}(.)} \quad \int_{0}^{t} \frac{1}{2} \log \left(1+p^{s c}(u)+p^{b}(u)\right) d u
$$

where $p^{s c}(u)$ and $p^{b}(u)$ are the powers drained from the SC and the battery during the $0 \leq u \leq t$ time interval. The energy constraints for (4) are: $\int_{0}^{t}\left(p^{s c}(u)+\epsilon^{s c}\right) d u \leq E^{s c}$ and $\int_{0}^{t}\left(p^{b}(u)+\epsilon^{b}\right) d u \leq \eta E^{b}$ where $\epsilon^{s c}+\epsilon^{b}=\epsilon$. We remark that the single epoch analysis in [14], [20] does not immediately apply to our problem since our problem involves two power variables and the transmitter incurs a processing cost when either one (or both) of these power variables is non-zero and the processing energy can be drained from two different energy storage devices.

We observe that due to the concavity of the $\log ($.$) function,$ $p^{s c}(u)+p^{b}(u)$ must remain constant whenever $p^{s c}(u)+$ $p^{b}(u)>0$ and such an allocation is always feasible since the energies $E^{s c}$ and $E^{b}$ are assumed to be available before the transmission starts. This, in turn, implies that the transmission duration $t$ is $t=\frac{E^{s c}+\eta E^{b}}{p^{s c}+p^{b}+\epsilon}$ where $p^{s c}$ and $p^{b}$ are constant powers drained from the SC and the battery during the $0 \leq u \leq t$ interval. Hence, the objective function in (4) is expressed as a single-variable function of $p^{s c}+p^{b}$ : $\frac{E^{s c}+\eta E^{b}}{p^{s c}+p^{b}+\epsilon} \frac{1}{2} \log \left(1+p^{s c}+p^{b}\right)$. Equating its derivative to zero, we obtain the following equation (c.f. [14], [20]):

$$
\frac{\log \left(1+p^{*}\right)}{\left(p^{*}+\epsilon\right)}=\frac{1}{1+p^{*}}
$$

Let $p^{*}$ be the solution of the equation in (5). Then, $p^{s c *}$ and $p^{b *}$ are the solutions of (4) if $p^{s c *}+p^{b *}=p^{*}$. Note that $p^{*}$ is the unique solution of (5), which parametrically depends on $\epsilon$ 
and is independent of $E^{s c}$ and $E^{b}$ [14], [20]. Moreover, we note that the selections of $p^{s c *}$ and $p^{b *}$ are not unique and they determine $\epsilon^{s c}$ and $\epsilon^{b}$. In particular, we have

$$
\begin{aligned}
\epsilon^{s c} & =\frac{E^{s c}}{E^{s c}+\eta E^{b}}\left(p^{*}+\epsilon\right)-p^{s c *} \\
\epsilon^{b} & =\frac{\eta E^{b}}{E^{s c}+\eta E^{b}}\left(p^{*}+\epsilon\right)-p^{b *}
\end{aligned}
$$

Now, let us impose a deadline $t \leq T$ to the problem in (4). If the deadline $T$ satisfies $T \geq \frac{E^{s c}+\eta E^{b}}{p^{*}+\epsilon}$, the solution is the same as the solution with an infinite deadline. On the other hand, if $T \leq \frac{E^{s c}+\eta E^{b}}{p^{*}+\epsilon}$, then $p^{s c *}+p^{b *}=\frac{E^{s c}+\eta E^{b}}{T}-\epsilon$ and $\epsilon^{s c}, \epsilon^{b}$ are determined as:

$$
\begin{aligned}
\epsilon^{s c} & =\frac{E^{s c}}{T}-p^{s c *} \\
\epsilon^{b} & =\frac{\eta E^{b}}{T}-p^{b *}
\end{aligned}
$$

In the infinite deadline case, one possible selection is $p^{s c *}=\frac{E^{s c}}{E^{s c}+\eta E^{b}} p^{*}$ and $p^{b *}=\frac{\eta E^{b}}{E^{s c}+\eta E^{b}} p^{*} ; \epsilon^{s c}$ and $\epsilon^{b}$ are determined according to (6)-(7). This selection facilitates an alternative view of the problem: If in the first $t^{s c}=\frac{E^{s c}}{p^{*}+\epsilon}$ time units, $p^{s c}=p^{*}, p^{b}=0$ and in the following $t^{b}=\frac{\eta E^{b}}{p^{*}+\epsilon}$ time units, $p^{b}=p^{*}$ and $p^{s c}=0$, the optimal throughput for (4) is achieved. Moreover, the processing energy is drained from the SC and the battery with power $\epsilon$ only when they are active. This selection has the following counterpart if the deadline is finite: When $\frac{E^{s c}}{p^{*}+\epsilon} \leq T \leq \frac{E^{s c}+\eta E^{b}}{p^{*}+\epsilon}, p^{s c}=p^{*}$ over the first $t^{s c}=\frac{E^{s c}}{p^{*}+\epsilon}$ time units and $p^{b}$ is determined by water-filling $\eta E^{b}$ units of energy over $[0, T]$ interval given $p^{s c}$ and no processing cost from the battery in the first $t^{s c}$ units. Secondly, if $T<\frac{E^{s c}}{p^{*}+\epsilon}, p^{s c}=\frac{E^{s c}}{T}-\epsilon$ and $p^{b}=\frac{\eta E^{b}}{T}$ over $[0, T]$.

This alternative view of the problem suggests that a solution for (4) can be found by solving

$$
\max _{t^{s c}, t^{b}, p^{s c}, p_{1}^{b}, p_{2}^{b}} \frac{t^{s c}}{2} \log \left(1+p^{s c}+p_{1}^{b}\right)+\frac{t^{b}}{2} \log \left(1+p_{2}^{b}\right)
$$

where the energy constraints are $t^{s c}\left(p^{s c}+\epsilon\right) \leq E^{s c}$ and $t^{s c} p_{1}^{b}+$ $t^{b}\left(p_{2}^{b}+\epsilon\right) \leq \eta E^{b}$ along with the deadline $t^{s c}+t^{b} \leq T$. Note that the processing energy is drained from the SC in the first $t^{s c}$ units and from the battery in the remaining time units. The problem (10) has a unique solution ${ }^{2} t^{s c *}, t^{b *}, p^{s c *}, p_{1}^{b *}, p_{2}^{b *}$. To see this, note that all of the time and energy constraints must be satisfied with equality and whenever $t^{b *}>0$, we must have $p^{s c *}+p_{1}^{b *}=p_{2}^{b *}=\frac{E^{s c}+\eta E^{b}}{T}-\epsilon$, which along with the time and energy constraints, determine the variables in (10) uniquely. Similarly, if $t^{b *}=0$, then $p^{s c *}=\frac{E^{s c}}{T}-\epsilon, p_{1}^{b *}=\frac{\eta E^{b}}{T}$ and $p_{2}^{b *}$ can be selected arbitrarily. Note that using the unique solution $t^{s c *}, t^{b *}, p^{s c *}, p_{1}^{b *}, p_{2}^{b *}$ of (10), we can get a solution of (4) by setting the SC power as $\frac{t^{s c *}}{t^{s c *}+t^{b *}} p^{s c *}$ and the battery power as $\frac{t^{s c *}}{t^{s c *}+t^{b *}} p_{1}^{b *}+\frac{t^{b *}}{t^{s c *}+t^{b *}} p_{2}^{b *}$. Moreover, $\frac{t^{s c *}}{t^{s c *}+t^{b *}} \epsilon$ units

${ }^{2}$ If $t^{b *}=0, p_{2}^{b *}$ can be selected arbitrarily; however, this does not violate the uniqueness of the solution. of processing energy is drained from the $\mathrm{SC}$ and the remaining processing energy is drained from the battery. We note that in an optimal solution of (10), $p_{1}^{b *}=0$ whenever $t_{i}^{s c *}+t_{i}^{b *}<T$.

\section{B. The Case of Multiple Epochs}

We now consider the multiple epoch case. As the rate-power relation is concave and the processing cost is additive and independent of the transmit power level, the transmit power policy $p(t)$ has to be constant during each epoch $i$ as long as $p(t)>0$; see also [14], [20]. Therefore, we get the following constraints for all $i$ :

$$
\begin{aligned}
\sum_{j=1}^{i}\left(\left(p_{j}^{s c}+\epsilon\right) t_{j}^{s c}+\delta_{j} t_{j}^{s c}\right) & \leq \sum_{j=0}^{i-1} E_{j}^{s c} \\
\sum_{j=0}^{i} E_{j}^{s c}-\sum_{j=1}^{i}\left(\left(p_{j}^{s c}+\epsilon\right) t_{j}^{s c}+\delta_{j} t_{j}^{s c}\right) & \leq E_{\max } \\
\sum_{j=1}^{i}\left(p_{1 j}^{b} t_{j}^{s c}+\left(p_{2 j}^{b}+\epsilon\right) t_{j}^{b}\right) & \leq \sum_{j=0}^{i-1}\left(\eta E_{j}^{b}+\eta \delta_{j} t_{j}^{s c}\right) \\
p_{i}^{s c}, p_{1 i}^{b}, p_{2 i}^{b}, \delta_{i} & \geq 0
\end{aligned}
$$

where $E_{i}^{s c}=\min \left(E_{i}, E_{\max }\right)$ and $E_{i}^{b}=\left(E_{i}-E_{\max }\right)^{+}$. We set $\delta_{0}=0$ and $\delta_{N}=0$ by convention. $t_{i}=t_{i}^{s c}+t_{i}^{b}$ is the time portion of epoch $i$ in which the transmitter is active. Thus, $0 \leq t_{i} \leq \ell_{i}$. We note that the constraint set in (11)-(14) is not convex. To circumvent this difficulty, we introduce a change of variables: $\alpha_{i} \triangleq p_{i}^{s c} t_{i}^{s c}, \beta_{i} \triangleq p_{2 i}^{b} t_{i}^{b}, \theta_{i} \triangleq p_{1 i}^{b} t_{i}^{s c}$ and $\gamma_{i} \triangleq \delta_{i} t_{i}^{s c}$. The constraint set in terms of the new variables is:

$$
\begin{aligned}
\sum_{j=1}^{i}\left(\alpha_{j}+\epsilon t_{j}^{s c}+\gamma_{j}\right) & \leq \sum_{j=0}^{i-1} E_{j}^{s c} \\
\sum_{j=0}^{i} E_{j}^{s c}-\sum_{j=1}^{i}\left(\alpha_{j}+\epsilon t_{j}^{s c}+\gamma_{j}\right) & \leq E_{\max } \\
\sum_{j=1}^{i}\left(\theta_{j}+\beta_{j}+\epsilon t_{j}^{b}\right) & \leq \sum_{j=0}^{i-1}\left(\eta E_{j}^{b}+\eta \gamma_{j}\right) \\
0 \leq t_{i}^{s c}+t_{i}^{b} & \leq \ell_{i} \\
t_{i}^{s c}, t_{i}^{b}, \alpha_{i}, \beta_{i}, \theta_{i}, \gamma_{i} & \geq 0
\end{aligned}
$$

Throughput maximization problem in the new variable set is:

$$
\begin{aligned}
\max _{\alpha_{i}, \beta_{i}, \theta_{i}, t_{i}^{s c}, t_{i}^{b}, \gamma_{i} \geq 0} & \sum_{i=1}^{N} \frac{t_{i}^{s c}}{2} \log \left(1+\frac{\alpha_{i}}{t_{i}^{s c}}+\frac{\theta_{i}}{t_{i}^{s c}}\right) \\
& +\frac{t_{i}^{b}}{2} \log \left(1+\frac{\beta_{i}}{t_{i}^{b}}\right) \\
\text { s.t. } \quad(15) & -(19)
\end{aligned}
$$

The concavity of the objective in (20) follows from the convexity preservation of the perspective operation [21] and the fact that $\frac{t_{i}}{2} \log \left(1+\frac{\alpha_{i}}{t_{i}}+\frac{\beta_{i}}{t_{i}}\right)$ is the perspective of the concave function $\frac{1}{2} \log \left(1+\alpha_{i}+\beta_{i}\right)$, c.f. [14]. The Lagrangian for (20) is: 


$$
\begin{aligned}
\mathcal{L}= & -\sum_{i=1}^{N}\left[\frac{t_{i}^{s c}}{2} \log \left(1+\frac{\alpha_{i}}{t_{i}^{s c}}+\frac{\theta_{i}}{t_{i}^{s c}}\right)+\frac{t_{i}^{b}}{2} \log \left(1+\frac{\beta_{i}}{t_{i}^{b}}\right)\right] \\
& +\sum_{i=1}^{N} \lambda_{i}\left[\sum_{j=1}^{i}\left(\alpha_{j}+\epsilon t_{j}^{s c}+\gamma_{j}\right)-\sum_{j=0}^{i-1} E_{j}^{s c}\right] \\
& +\sum_{i=1}^{N-1} \mu_{i}\left[\sum_{j=0}^{i} E_{j}^{s c}-\sum_{j=1}^{i}\left(\alpha_{j}+\epsilon t_{j}^{s c}+\gamma_{j}\right)-E_{\max }\right] \\
& +\sum_{i=1}^{N} \nu_{i}\left[\sum_{j=1}^{i}\left(\theta_{i}+\beta_{i}+\epsilon t_{j}^{b}\right)-\sum_{j=0}^{i-1}\left(\eta E_{j}^{b}+\eta \gamma_{j}\right)\right] \\
& -\sum_{i=1}^{N} \rho_{1 i} \alpha_{i}-\sum_{i=1}^{N} \rho_{2 i} \theta_{i}-\sum_{i=1}^{N} \rho_{3 i} \beta_{i}-\sum_{i=0}^{N} \xi_{i} \gamma_{i} \\
& -\sum_{i=1}^{N} \sigma_{1 i} t_{i}^{s c}-\sum_{i=1}^{N} \sigma_{2 i} t_{i}^{b}+\sum_{i=1}^{N} z_{i}\left(t_{i}^{s c}+t_{i}^{b}-\ell_{i}\right)
\end{aligned}
$$

where $\lambda_{i}, \mu_{i}, \nu_{i}, \rho_{1 i}, \rho_{2 i}, \rho_{3 i}, \xi_{i}, \sigma_{1 i}, \sigma_{2 i}$ and $z_{i}$ are the Lagrange multipliers. The KKT optimality conditions for (20) are:

$$
\begin{array}{r}
-\frac{t_{i}^{s c}}{t_{i}^{s c}+\alpha_{i}+\theta_{i}}+\sum_{j=i}^{N} \lambda_{j}-\sum_{j=i}^{N-1} \mu_{j}-\rho_{1 i}=0 \\
-\frac{t_{i}^{s c}}{t_{i}^{s c}+\alpha_{i}+\theta_{i}}+\sum_{j=i}^{N} \nu_{j}-\rho_{2 i}=0 \\
-\frac{t_{i}^{b}}{t_{i}^{b}+\beta_{i}}+\sum_{j=i}^{N} \nu_{j}-\rho_{3 i}=0 \\
\sum_{j=i}^{N} \lambda_{j}-\sum_{j=i}^{N-1} \mu_{j}-\eta \sum_{j=i+1}^{N} \nu_{j}-\xi_{i}=0 \\
\frac{\alpha_{i}+\theta_{i}}{t_{i}^{s c}+\alpha_{i}+\theta_{i}}-\log \left[\frac{t_{i}^{s c}+\alpha_{i}+\theta_{i}}{t_{i}^{s c}}\right] \\
+\epsilon\left[\sum_{j=i}^{N} \lambda_{j}-\sum_{j=i}^{N-1} \mu_{j}\right]-\sigma_{1 i}+z_{i}=0 \\
\frac{\beta_{i}}{t_{i}^{b}+\beta_{i}}-\log \left[\frac{t_{i}^{b}+\beta_{i}}{t_{i}^{b}}\right]+\epsilon \sum_{j=i}^{N} \nu_{j}-\sigma_{2 i}+z_{i}=0
\end{array}
$$

and the corresponding complementary slackness conditions:

$$
\begin{aligned}
\lambda_{i}\left[\sum_{j=1}^{i}\left(\alpha_{j}+\epsilon t_{j}^{s c}+\gamma_{j}\right)-\sum_{j=0}^{i-1} E_{j}^{s c}\right] & =0 \\
\mu_{i}\left[\sum_{j=0}^{i} E_{j}^{s c}-\sum_{j=1}^{i}\left(\alpha_{j}+\epsilon t_{j}^{s c}+\gamma_{j}\right)-E_{\max }\right] & =0 \\
\nu_{i}\left[\sum_{j=1}^{i}\left(\theta_{j}+\beta_{j}+\epsilon t_{j}^{b}\right)-\sum_{j=0}^{i-1}\left(\eta E_{j}^{b}+\eta \gamma_{j}\right)\right] & =0 \\
\rho_{1 i} \alpha_{i}=\rho_{2 i} \theta_{i}=\rho_{3 i} \beta_{i}=\xi_{i} \gamma_{i} & =0
\end{aligned}
$$

$$
\sigma_{1 i} t_{i}^{s c}=\sigma_{2 i} t_{i}^{b}=z_{i}\left(t_{i}^{s c}+t_{i}^{b}-\ell_{i}\right)=0
$$

We remark that the optimization problem in (20) may have many solutions. Based on our analysis of a single epoch, we observe some properties of an optimal solution for (20) in the following lemmas.

Lemma 1 If $0<t_{i}^{s c *}<\ell_{i}$, then $\delta_{i}^{*}=0$.

Proof: For the case when $t_{i}^{b *}=0$, we have $p_{1 i}^{b *}=p_{2 i}^{b *}=0$. Hence, $\sigma_{1 i}=0, z_{i}=0$ and $\nu_{i}=0$. By (26), $\frac{\log \left(1+p_{i}^{c *}\right)}{p_{i}^{c *}+\epsilon}=$ $\frac{1}{1+p_{i}^{s c *}}$ and therefore $p_{i}^{s c *}=p^{*}$. By (22) and (25), we get $\xi_{i}>0$ and hence $\gamma_{i}=0$ and $\delta_{i}^{*}=0$.

When $t_{i}^{b *}>0$, we have $p_{2 i}^{b *}>0$. From the slackness conditions in (31)-(32), $\sigma_{1 i}=\sigma_{2 i}=\rho_{1 i}=\rho_{2 i}=0$. By (22), $\sum_{j=i}^{N} \lambda_{j}-\sum_{j=i}^{N-1} \mu_{j}=\frac{1}{1+p_{i}^{s c *}}$. By (23), $\sum_{j=i}^{N} \nu_{j}=\frac{1}{1+p_{2 i}^{b *}}=$ $\frac{1}{1+p_{i}^{s c *}}$. Using this in (25), we have $\xi_{i}=(1-\eta)\left(\frac{1}{1+p_{i}^{s c *}}\right)+$ $\eta \nu_{i}$. By (30), $\nu_{i} \geq 0$ and hence $\xi_{i}>0$ and together with the slackness condition $\xi_{i} \gamma_{i}=0$, we get $\delta_{i}^{*}=0$.

Lemma 2 If $t_{i}^{s c *}+t_{i}^{b *}=\ell_{i}$ and $p_{1 i}^{b *} \neq 0$, then $\delta_{i}^{*}=0$.

Proof: Note that $t_{i}^{s c *}>0$ as energy is first allocated to the SC. Hence, $p_{i}^{s c *}>0$ and $p_{1 i}^{b *}>0$. By the slackness condition in (31), $\rho_{1 i}=\rho_{2 i}=0$. From (22)-(23), we have $\sum_{j=i}^{N} \lambda_{j}-$ $\sum_{j=i}^{N-1} \mu_{j}=\frac{1}{1+p_{i}^{s c *}+p_{1 i}^{b *}}=\sum_{j=i}^{N} \nu_{j}$. Using this in (25), we have $\xi_{i}=(1-\eta)\left(\frac{1}{1+p_{i}^{s c *}+p_{1 i}^{b *}}\right)+\eta \nu_{i}>0$ as $\nu_{i} \geq 0$ and $0<\eta<1$. This, from the corresponding slackness condition, implies $\gamma_{i}=0$. As $t_{i}^{s c *}>0$, we get $\delta_{i}^{*}=0$.

Lemma 3 If $t_{i}^{s c *}+t_{i}^{b *}=\ell_{i}, t_{i}^{b *} \neq 0$ and $p_{1 i}^{b *} \neq 0$, then $p_{i}^{s c *}+p_{1 i}^{b *}=p_{2 i}^{b *} \geq p^{*}$.

Proof: By (31), we have $\rho_{1 i}=\rho_{2 i}=\rho_{3 i}=0$. From (23)-(24), we have $\sum_{j=i}^{N} \nu_{i}=\frac{1}{1+p_{i}^{s c *}+p_{1 i}^{b *}}=\frac{1}{1+p_{2 i}^{b *}}$. The second equality will be satisfied only when $p_{i}^{s c *}+p_{1 i}^{b *}=p_{2 i}^{b *}$. Next, since $t_{i}^{s c *}+t_{i}^{b *}=\ell_{i}$, from the slackness condition in (32), $z_{i} \geq 0$. Also, from (22), we have $\sum_{j=i}^{N} \lambda_{i}-\sum_{j=i}^{N-1} \mu_{i}=\frac{1}{1+p_{i}^{s *}+p_{1 i}^{b *}}$. Combining this with (26), and by the fact that $z_{i} \geq 0$, we get $\log \left(1+p_{i}^{s c *}+p_{1 i}^{b *}\right) \geq \frac{\epsilon+p_{i}^{s c *}+p_{1 i}^{b *}}{1+p_{i}^{s c *}+p_{1 i}^{b *}}$, which holds only when $p_{i}^{s c *}+p_{1 i}^{b *} \geq p^{*}$ where $p^{*}$ is the threshold power level.

Lemmas 1-3 provide useful properties of the optimal power allocation in the presence of additive processing cost $\epsilon$. In particular, we first determine a threshold power level $p^{*}$ based only on $\epsilon$, and determine the energy flow in time accordingly. In view of these properties, we continue our analysis for fixed $\delta_{i}=0$ case in the following section.

\section{Optimal Policy for Fixed $\delta_{i}=0$}

For fixed $\delta_{i}=0$, the problem is the following: 


\section{CONCLUSION}

$\max _{\alpha_{i}, \beta_{i}, \theta_{i}, t_{i}^{s c}, t_{i}^{b} \geq 0} \sum_{i=1}^{N} \frac{t_{i}^{s c}}{2} \log \left(1+\frac{\alpha_{i}}{t_{i}^{s c}}+\frac{\theta_{i}}{t_{i}^{s c}}\right)+\frac{t_{i}^{b}}{2} \log \left(1+\frac{\beta_{i}}{t_{i}^{b}}\right)$

$$
\begin{array}{ll}
\text { s.t. } & (15)-(19) \\
& \delta_{i}=0, \quad \forall i
\end{array}
$$

We next show that the solution of (33) is found by applying the directional glue-pouring algorithm in [14] only twice.

Lemma 4 For fixed $\delta_{i}=0$, let $\hat{p}_{i}^{s c}$ and $\hat{t}_{i}^{s c}$ be the outcome of directional glue-pouring given $p_{i}^{b}=0$. Let $\hat{p}_{1 i}^{b}, \hat{p}_{2 i}^{b}$ and $\hat{t}_{i}^{b}$ be the outcome of directional glue-pouring given $\hat{p}_{i}^{\text {sc }}$ and no processing cost from the battery over the first $\hat{t}_{i}^{s c}$ time units. Then, $\hat{p}_{i}^{s c}, \hat{p}_{1 i}^{b}, \hat{p}_{2 i}^{b}, \hat{t}_{i}^{s c}$ and $\hat{t}_{i}^{b}$ are jointly optimal for (33).

The proof of Lemma 4 is skipped here due to space limitations; see [22] for a detailed proof.

\section{Determining the Optimal $\delta_{i}^{*}$}

In the previous section, we have seen that for $\hat{p}_{i}^{s c}, \hat{p}_{1 i}^{b}, \hat{p}_{2 i}^{b}, \hat{t}_{i}^{s c}$ and $\hat{t}_{i}^{b}$, there exist Lagrange multipliers $\lambda_{i}, \mu_{i}, \nu_{i}, \rho_{1 i}, \rho_{2 i}$ and $\rho_{3 i}$ that satisfy (22)-(27); however, it is not clear if there exist $\xi_{i}$ that satisfy (25). In this section, we propose a method to update $\hat{p}_{i}^{s c}, \hat{p}_{1 i}^{b}, \hat{p}_{2 i}^{b}, \hat{t}_{i}^{s c}, \hat{t}_{i}^{b}$ and the corresponding Lagrange multipliers so that we obtain $\xi_{i}$ and $\delta_{i}^{*}$ such that (22)-(27) are satisfied. For brevity and clarity of explanation, we assume without loss of generality that $p_{i}^{s c}$ is higher than the threshold level $p^{*}$ for $i=1$ and equal to $p^{*}$ for $i=2, \ldots, N$.

By Lemmas 1 and $2, \delta_{i}^{*}=0$ if $t_{i}^{s c *}<\ell_{i}$ or $p_{1 i}^{b *}>0$. Indeed, $\delta_{i}>0$, only if $\hat{p}_{i}^{s c}>p^{*}$. Thus, we first consider to update the values of $z_{i}$ for those epochs where $z_{i}=0$. The energy for these epochs comes from those previous epochs where $z_{i} \geq 0$ and $p_{1 i}^{b *}=0$.

In order to find $\delta_{i}^{*}$, we transform the energy and water levels. We set the bottom levels of epochs with $\hat{p}_{i}^{s c}>p^{*}$ and for the remaining epochs, we set the bottom level to $\frac{1}{\eta}$ and multiply the water level by $\frac{1}{\eta}$. In this transformed setting, if the water level is higher in an epoch where $\hat{p}_{i}^{s c}>p^{*}$ compared to the next epoch, then we transfer $\delta_{i}$ units of water from the SC in this epoch and $\frac{1}{n} \delta_{i}$ units of water is added to the battery in the next epoch. This way, we transfer the energy in a systematic way. In the particular case when $p_{1}^{s c}>p^{*}$ and $p_{i}^{s c}=p^{*}$ for $i=2, \ldots, N$, energy is transferred from epoch 1 to epochs $i=2, \ldots, N$. Note that $z_{1}>0$ and $z_{i}=0$ for $i=2, \ldots, N$ for this particular allocation. When energy is transferred, $\lambda_{i}, \mu_{i}$ are increased and $\nu_{i}$ remains unchanged until $\hat{t}_{i}^{s c}+t_{i}^{b}=\ell_{i}$ provided that sufficiently large energy is transferred. If the water level in epoch 1 is still higher than $p^{*}$, we start transferring energy to the next epoch in the transformed setting. We also note that the transferred energy can be utilized in later epochs as long as the power is kept at $p^{*}$ and hence the optimal allocation is not unique. Once $t_{i}^{s c}+t_{i}^{b}=\ell_{i}$, we have to make $z_{i}>0$ due to the slackness condition in (31) and raise the transmit power levels $p_{1 i}^{b}$ above zero and $p_{2 i}^{b}$ above the threshold level $p^{*}$.
We study offline throughput maximization in an energy harvesting transmitter with hybrid energy storage and non-zero processing power. In contrast to previous work, an additional challenge arises as the processing power is drained from two energy storage units. We show that the solution is obtained by a sequential application of directional glue-pouring algorithm.

\section{REFERENCES}

[1] V. Raghunathan, A. Kansal, J. Hsu, J. K. Friedman, and M. B. Srivastava, "Design considerations for solar energy harvesting wireless embedded systems," in IEEE IPSN, pp. 457-462, April 2005.

[2] J. Yang and S. Ulukus, "Optimal packet scheduling in an energy harvesting communication system," IEEE Trans. Comm., vol. 60, pp. 220-230, January 2012

[3] K. Tutuncuoglu and A. Yener, "Optimum transmission policies for battery limited energy harvesting nodes," IEEE Trans. Wireless Comm., vol. 11, pp. 1180-1189, March 2012.

[4] O. Ozel, K. Tutuncuoglu, J. Yang, S. Ulukus, and A. Yener, “Transmission with energy harvesting nodes in fading wireless channels: Optimal policies," IEEE JSAC, vol. 29, pp. 1732-1743, September 2011.

[5] J. Yang, O. Ozel, and S. Ulukus, "Broadcasting with an energy harvesting rechargeable transmitter," IEEE Trans. Wireless Comm., vol. 11, pp. 571-583, February 2012.

[6] O. Ozel, J. Yang, and S. Ulukus, "Optimal broadcast scheduling for an energy harvesting rechargeable transmitter with a finite capacity battery," IEEE Trans. Wireless Comm., vol. 11, pp. 2193-2203, June 2012.

[7] J. Yang and S. Ulukus, "Optimal packet scheduling in a multiple access channel with energy harvesting transmitters," Jour. of Comm. and Netw., vol. 14, pp. 140-150, April 2012.

[8] K. Tutuncuoglu and A. Yener, "Sum-rate optimal power policies for energy harvesting transmitters in an interference channel," Jour of Comm. and Netw., vol. 14, pp. 151-161, April 2012.

[9] C. Huang, R. Zhang, and S. Cui, "Throughput maximization for the Gaussian relay channel with energy harvesting constraints," IEEE JSAC, vol. 31, pp. 1469-1479, August 2013.

[10] D. Gunduz and B. Devillers, "Two-hop communication with energy harvesting," in IEEE CAMSAP, December 2011.

[11] B. Devillers and D. Gunduz, "A general framework for the optimization of energy harvesting communication systems with battery imperfections," Jour. of Comm. and Netw., vol. 14, pp. 130 - 139, Apr. 2012.

[12] B. Gurakan, O. Ozel, J. Yang, and S. Ulukus, "Energy cooperation in energy harvesting communications," IEEE Trans. Comm. To appear.

[13] J. Xu and R. Zhang, "Throughput optimal policies for energy harvesting wireless transmitters with non-ideal circuit power," IEEE JSAC. To appear.

[14] O. Orhan, D. Gunduz, and E. Erkip, "Throughput maximization for an energy harvesting system with processing cost," in IEEE ITW, September 2012.

[15] K. Tutuncuoglu and A. Yener, "Communicating using an energy harvesting transmitter: Optimum policies under energy storage losses," IEEE Trans. Wireless Comm., submitted August 2012, [arXiv:1208.6273].

[16] O. Ozel, K. Shahzad, and S. Ulukus, "Optimal scheduling for energy harvesting transmitters with hybrid energy storage," in IEEE ISIT, July 2013.

[17] S. Cui, A. J. Goldsmith, and A. Bahai, "Energy-constrained modulation optimization," IEEE Trans. Wireless Comm., vol. 4, pp. 2349-2360, September 2005.

[18] Q. Bai and J. A. Nossek, "Throughput maximization for energy harvesting nodes with generalized circuit power modeling," in IEEE SPAWC, June 2012.

[19] M. Gregori and M. Payaro, "Throughput maximization for a wireless energy harvesting node considering the circuit power consumption," in IEEE VTC, September 2012.

[20] P. Youssef-Massaad, L. Zheng, and M. Medard, "Bursty transmission and glue pouring: On wireless channels with overhead costs," IEEE Trans. Wireless Comm., vol. 7, pp. 5188-5194, December 2008.

[21] S. Boyd and L. Vandenberghe, Convex Optimization. United Kingdom: Cambridge University Press, 2004.

[22] K. Shahzad, "Scheduling in energy harvesting systems with hybrid energy storage," MSc. Thesis, UMD, Sep. 2013. 